\title{
Changing your sex changes your brain: influences of testosterone and estrogen on adult human brain structure
}

\author{
Hilleke E Hulshoff Pol, Peggy T Cohen-Kettenis ${ }^{1}$, Neeltje E M Van Haren, Jiska S Peper, Rachel G H Brans, \\ Wiepke Cahn, Hugo G Schnack, Louis J G Gooren² and René S Kahn \\ Department of Psychiatry, Rudolf Magnus Institute of Neuroscience, University Medical Center Utrecht, A01.126, Heidelberglaan 100, 3584 CX Utrecht, \\ The Netherlands, ${ }^{1}$ Department of Medical Psychology and ${ }^{2}$ Department of Endocrinology, VU University Medical Center, Amsterdam, The Netherlands \\ (Correspondence should be addressed to H E Hulshoff Pol; Email: h.e.hulshoff@azu.nl)
}

\begin{abstract}
Objective: Sex hormones are not only involved in the formation of reproductive organs, but also induce sexually-dimorphic brain development and organization. Cross-sex hormone administration to transsexuals provides a unique possibility to study the effects of sex steroids on brain morphology in young adulthood.

Methods: Magnetic resonance brain images were made prior to, and during, cross-sex hormone treatment to study the influence of anti-androgen + estrogen treatment on brain morphology in eight young adult male-to-female transsexual human subjects and of androgen treatment in six femaleto-male transsexuals.

Results: Compared with controls, anti-androgen + estrogen treatment decreased brain volumes of male-to-female subjects towards female proportions, while androgen treatment in female-to-male subjects increased total brain and hypothalamus volumes towards male proportions.

Conclusions: The findings suggest that, throughout life, gonadal hormones remain essential for maintaining aspects of sex-specific differences in the human brain.
\end{abstract}

European Journal of Endocrinology 155 S107-S114

\section{Introduction}

Transsexualism is the condition in which a person with apparently normal somatic sexual differentiation of one sex is convinced that he or she is actually a member of the opposite sex. This sense is so pronounced and persistent that transsexuals seek treatment to, as far as medically possible, physically change their bodies from male into female or vice versa. Prior to surgical sex reassignment, transsexuals receive treatment with cross-sex hormones. Male-to-female transsexuals (MFs) are treated with estrogens and anti-androgens (to suppress the production and biological effects of circulating androgens) and female-to-male transsexuals (FMs) are treated with androgens (in FMs, androgens, without additional hormone treatment, usually suppress menstruation; circulating estrogens are not substantially reduced as a result of peripheral aromatization of administered androgens). There is no known fundamental difference in sensitivity to the biological action of sex steroids on the basis of genetic configurations or gonadal status. Thus,

This paper was presented at the 4th Ferring Pharmaceuticals International Paediatric Endocrinology Symposium, Paris (2006). Ferring Pharmaceuticals has supported the publication of these proceedings. in transsexuals, the influence of cross-sex hormones can be studied relatively independent of their original endocrine status as male or female.

It is well established in mammals that differences in male and female brain structures can be reversed by sex hormones, even in adulthood (1). However, it is not known whether alterations in sex hormone levels can change structures of the human brain in adulthood. In human adults, the volumes of the brain and hypothalamus of males tend to be larger than those of females (2). The preoptic nucleus of the hypothalamus is even twice as large in males as in females (3). Moreover, in some studies, when comparing the fractions of gray and white matter in the brain, adult females as compared with males were found to have a higher fraction of gray matter, whereas adult males as compared with females had a higher fraction of white matter $(4,5)$.

In rodents, brain differences between the sexes supposedly reflect differential exposure to sex hormones during perinatal brain development (6). Typically, perinatal exposure to high levels of testosterone results in male brain structure and in the absence of androgen exposure, female brain structure develops. In humans, testosterone probably exerts its masculinizing influence on the brain during prenatal development (7). However, we hypothesize that, in addition, circulating sex 
hormones in adulthood are required for the maintenance of sex differences in the human brain.

A few studies on brain structure in transsexuals have been conducted in post-mortem tissue. The bed nucleus of the stria terminalis of the hypothalamus, larger in males than in females, was found to be of female size in six MFs $(8,9)$ and of male size in one FM (9). All these transsexuals had received cross-sex hormone treatment before their brains were studied. Therefore, the altered size of the bed nucleus of the stria terminalis could have been due to the exposure of cross-sex hormones in adult life. Alternatively, the different size of the bed nucleus of the stria terminalis in transsexuals could have been present prior to cross-sex hormones treatment, reflecting (potentially hormonally determined) differences in the development of the (pre- and perinatal) brain, or possibly genetic differences, between transsexuals and non-transsexuals (10). The aim of our study was to examine the influence of exposure to high levels of cross-sex hormones on brain structures in adulthood.

\section{Methods}

\section{Subjects}

Eight MFs and six FMs were recruited through the Outpatient Clinic from the Department of Psychiatry, University Medical Center Utrecht, The Netherlands, and through the Department of Endocrinology, VU University Medical Center, Amsterdam, The Netherlands, and compared with nine male and six female healthy comparison subjects (Table 1). Subjects signed informed consent after full explanation of the study. Inclusion criteria for transsexual patients were DSM-IV criteria for gender identity disorder, referral for hormone treatment, no severe medical illness, and age between 16 and 50 years. The diagnosis of gender identity disorder was made according to the structured clinical interview for DSM-IV axis-I disorders (11). Patients participated in the study after it was decided that they were eligible for hormone treatment. This decision was made according to the clinical protocols for the diagnosis and treatment of transsexuals of the University Medical Center Utrecht and VU University Medical Center, Amsterdam (12). Healthy comparison subjects were included from the structural neuroimaging database at the Department of Psychiatry, University Medical Center, Utrecht; following full explanation of all procedures, subjects signed informed consent. The healthy comparison subjects were matched to the transsexuals for age and original sex at first measurement. In addition, years of education - defined as the total number of years of education that was successfully completed - and scan interval between the first and the second magnetic resonance imaging scan acquisition in days was measured in each group. The Medical Ethical Committee for Human Subjects at the University Medical Center Utrecht approved the study.

\section{Brain image acquisition}

Magnetic resonance T1- and T2-weighted images were acquired on a Philips NT scanner operating at $1.5 \mathrm{~T}$ in all subjects. A three-dimensional-fast field echo (echo time $(\mathrm{TE})=4.6 \mathrm{~ms}$, repetition time $(\mathrm{TR})=30 \mathrm{~ms}$, flip angle $=30^{\circ}$, field of view $(\mathrm{FOV})=256 \times 256 \mathrm{~mm}^{2}$ ) with 160-180 contiguous coronal $1.2 \mathrm{~mm}$ slices and a T2weighted dual echo-turbo spin echo (TE1 $=14 \mathrm{~ms}$, $\mathrm{TE} 2=80 \mathrm{~ms}$, TR $=6350 \mathrm{~ms}$, flip angle $=90^{\circ}$, FOV $=$ $256 \times 256 \mathrm{~mm}^{2}$ ) with 120 contiguous coronal $1.6 \mathrm{~mm}$ slices of the whole head were used for the quantitative measurements. In addition, a T2-weighted dual echoturbo spin echo $(\mathrm{TE} 1=9 \mathrm{~ms}$, TE2 $=100 \mathrm{~ms}$, flip angle $=$ $90^{\circ}, \mathrm{FOV}=250 \times 250 \mathrm{~mm}^{2}$ ) with 17 axial $5 \mathrm{~mm}$ slices and $1.2 \mathrm{~mm}$ gap of the whole head was acquired for clinical neurodiagnostic evaluation.

\section{Image processing}

Processing was done on Hewlett Packard UNIX 9000 workstations and conventional Linux Personal Computers. All images were coded to ensure blindness for subject identification and diagnosis; scans were put into Talairach frame (no scaling) and corrected for inhomogeneities in the magnetic field. Quantitative assessments of the intracranial, total brain, gray and white matter of the cerebrum (total brain excluding cerebellum and stem), lateral and third ventricles, and peripheral cerebrospinal fluid (CSF) volumes were performed based on the histogram analyses and a series of mathematical morphology operators to connect all voxels of interest, as implemented and validated previously (13, 14; www.smri.nl). The interrater reliability of the automated volume measurements determined by the intraclass correlation coefficient in ten brains was 0.95 and higher.

Hypothalamus segmentation was done in coronal slices according to Nieuwenhuys et al. (15). The anterior boundary was the first coronal slice posterior of the anterior commissure $(\mathrm{AC})$, where the $\mathrm{AC}$ is no longer continuous and does not run through the emerging hypothalamus. Posterior, the last slice is where the mamillary bodies (excluded) end in the mid-sagittal slices - sagittal section is where the mamillary bodies are completed. Inferior, the hypothalamus ends where optic chiasma, infundibulum, and mamillary bodies begin. Superior, the AC-PC plane was used, which itself was not included in the hypothalamus. Lateral, the segmentation was limited by white matter. The intrarater reliability determined by the intraclass correlation coefficient in ten brains was 0.86 .

\section{Statistical analysis}

Data were examined for outliers, extreme values, and normality of distribution. There was no need for transformations. To investigate differences and changes 
over time in brain volumes between groups, multiple general linear modeling for repeated measures analyses were done for each of the volume measures (i.e. whole brain, hypothalamus, cerebral gray and white matter, and lateral and third ventricles), with group (transsexuals, comparisons), sex (male, MF, female, FM) as between-subject factors, and time (scan 2 minus scan 1) as within-subject factors. Age and intracranial volume at first measurement were added as covariates. In case of a significant finding, years of education and scan interval were also added to the statistical analysis as covariates to assess their possible influence.

\section{Results}

The influence of sex hormone treatment on brain morphology in young adult humans between 16 and 45 years of age (on average 25 years) was measured in eight MF and six FM human subjects (Table 1). Highresolution magnetic resonance brain images were obtained prior to and during cross-sex hormone treatment after a 4-month interval and prior to sex reassignment surgery (Fig. 1). MFs received dual therapy: the anti-androgen cyproterone acetate (Androcur) ( $2 \times 50 \mathrm{mg} /$ day $)$, having anti-androgenic properties both by its progestational action and competing for the androgen receptor, and a synthetic oral estrogen ethinylestradiol $(2 \times 50 \mu \mathrm{g} /$ day $)$. The estrogen doses used are 2-3 times more biopotent than traditional estrogen replacement in women. FMs received parenteral testosterone esters $(250 \mathrm{mg} / 2$ weeks i.m.). The testosterone doses were comparable to those used for testosterone replacement in hypogonadal males. Intracranial, whole brain, gray and white matter of the cerebrum, hypothalamus, and lateral and third ventricle volume changes in the transsexuals were compared with brain volume changes in nine male and six female healthy comparison subjects over a 1-year interval. The comparison subjects were matched with the transsexuals for age and original sex at first measurement. Years of education and scan interval differed between transsexuals and comparisons; in case of a significant finding, they were added to the statistical analysis as covariates. Note that the scan interval was longer in comparisons than in transsexuals; thus, group-by-time interaction effects for the influence of testosterone and estrogen were considered relatively conservative.

General linear modeling for repeated measures, correcting for age and intracranial volume, revealed significant group (transsexuals, comparisons) $\times$ sex (male, MF, female, FM) $\times$ time (scan 2 minus scan 1) interactions for whole brain $(F(1,23)=9.26, P=0.006)$ and hypothalamus $(F(1,23)=5.36, P=0.03)$ volumes (Table 1, Fig. 2). The significant group $\times$ sex $\times$ time effects were due to a decrease in hypothalamus and total brain volume in MFs treated with anti-androgens + estrogens

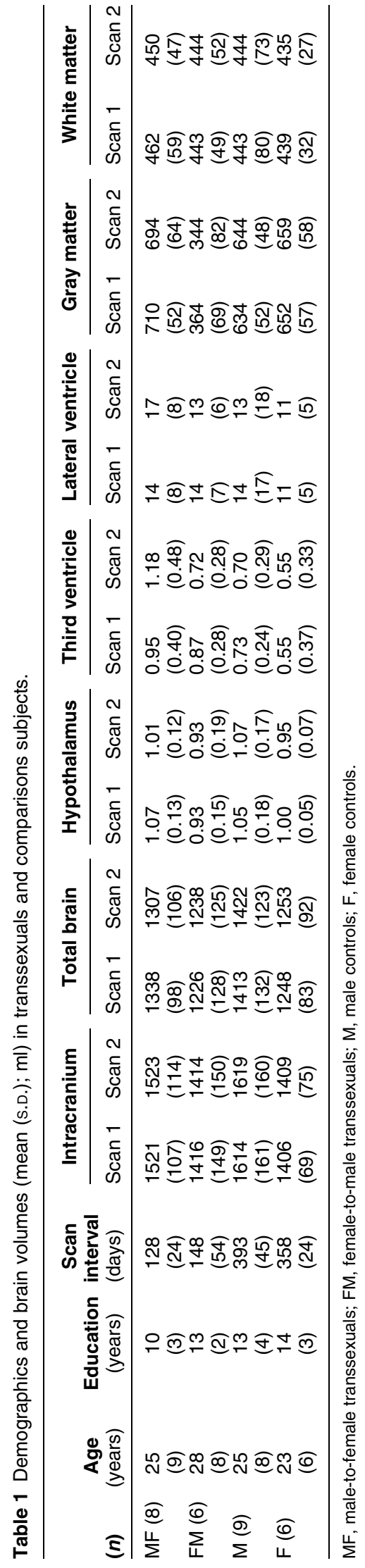

www.eje-online.org 


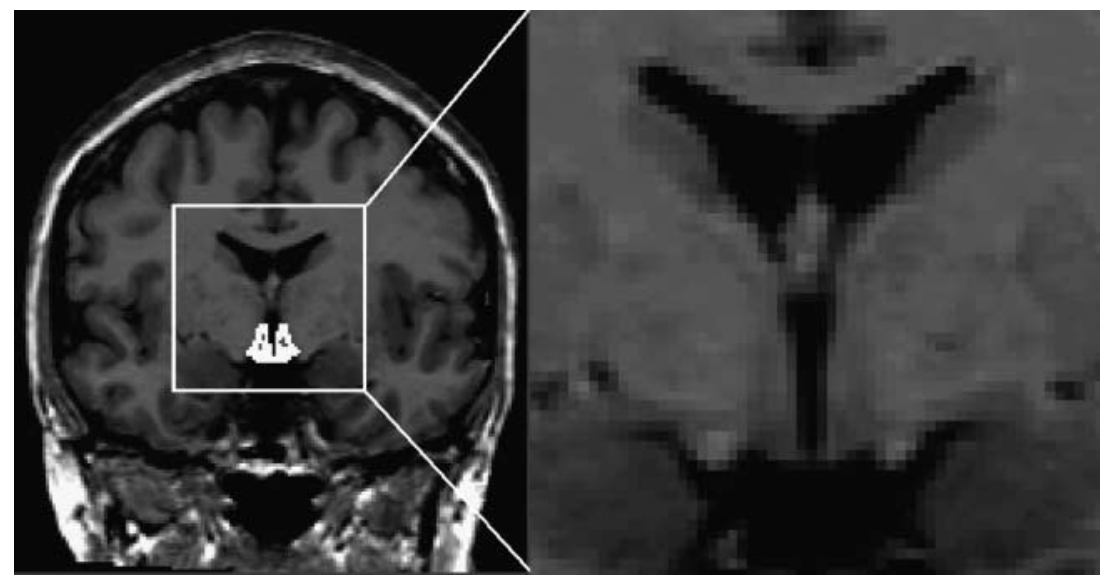

Figure 1 Magnetic resonance image acquisition of the whole-head coronal slice. Segmentation of the hypothalamus is shown in white. (suppressing testosterone virtually totally) after a 4-month interval compared with male comparison subjects, who showed a small increase. In addition, these effects were due to a lack of change in hypothalamic volume in testosterone-treated FMs after a 4-month interval, whereas it decreased in female comparison subjects. Moreover, testosterone administration to FMs increased total brain volume, whereas it remained unchanged in female comparison subjects.

Moreover, testosterone administration to FMs increased total brain volume, whereas it remained unchanged in female comparison subjects. Significant group $\times \operatorname{sex} \times$ time interactions were also found for third $(F(1,23)=$ 16.56, $P<0.0001)$ and lateral $(F(1,23)=21.90, P<$ $0.0001)$ ventricle volumes. The effects were due to increases in ventricle volumes in MFs as compared with a lack of change in third ventricle volume and subtle increase in lateral ventricle volume in male comparison subjects. Moreover, the findings were due to decreases in third and lateral ventricle volumes in FMs as compared with no change or subtle increases in volume in female comparison subjects. No significant effects were found for overall cerebral gray and white matter volumes.

In the young adult control subjects, no significant changes in volumes over time were found, except for an increase in lateral ventricle volume irrespective of sex $(F(1,11)=5.67, \quad P=0.036)$. Intracranial volume showed a small (and insignificant) increase in volume of $0.2 \%$. Although not significant, hypothalamus volume in female controls decreased over time.

As expected, at first measurement, intracranial volume was significantly larger in males as compared with females irrespective of the condition of transsexualism $(F(1,24)=10.10, P=0.004)$. No differences in intracranial volume were found between transsexuals and comparisons. After controlling for intracranial volume at initial measurement, no significant differences between the sexes or between transsexuals and control subjects were found for any of the brain volume measures. The relative proportion of white matter to gray matter was somewhat larger in the control females and the relative proportion of gray matter was somewhat larger in the control males, whereas the opposite was found for the transsexuals. However, this finding did not reach statistical significance. Third ventricle volume was larger in the transsexuals than in the controls, although not significant (main effect for group: $F(1,23)=3.36$, $P=0.08$; Table 1, Fig. 3). Thus, it is unlikely that the sexual dimorphic effects of sex hormone treatment on brain volumes over time were due to initial differences between groups or sexes. Moreover, it is also unlikely that level of education or scan interval influenced the results. Adding these factors to the analyses did not alter the results.

\section{Discussion}

The findings suggest that treatment of MFs with estrogens and anti-androgens decreases the male brain size towards female proportions, whereas treatment of FMs with androgens (not substantially affecting circulating estrogen levels) increases the female brain size towards male proportions. The magnitude of this change (i.e. $31 \mathrm{ml}$ over a 4-month period) is striking, since it signifies a decrease in brain volume, which is at least ten times the average decrease of around $2.5 \mathrm{ml}$ per year in healthy adults (16). Moreover, please note that consistent with the findings in the young adult control subjects of the present study, the longitudinal brain volume changes in young adults are normally small as compared with the older adults (17). The changes in total brain and hypothalamus volumes following cross-sex hormone treatment in the transsexuals were mirrored by changes in their third and lateral ventricle volumes, i.e. treatment with estrogens and anti-androgens in MFs increased third and lateral ventricle volumes, whereas treatment with androgens decreased the third and lateral ventricle volumes in FMs. This suggests that the total brain volume changes are at least in part due to changes in medial brain 

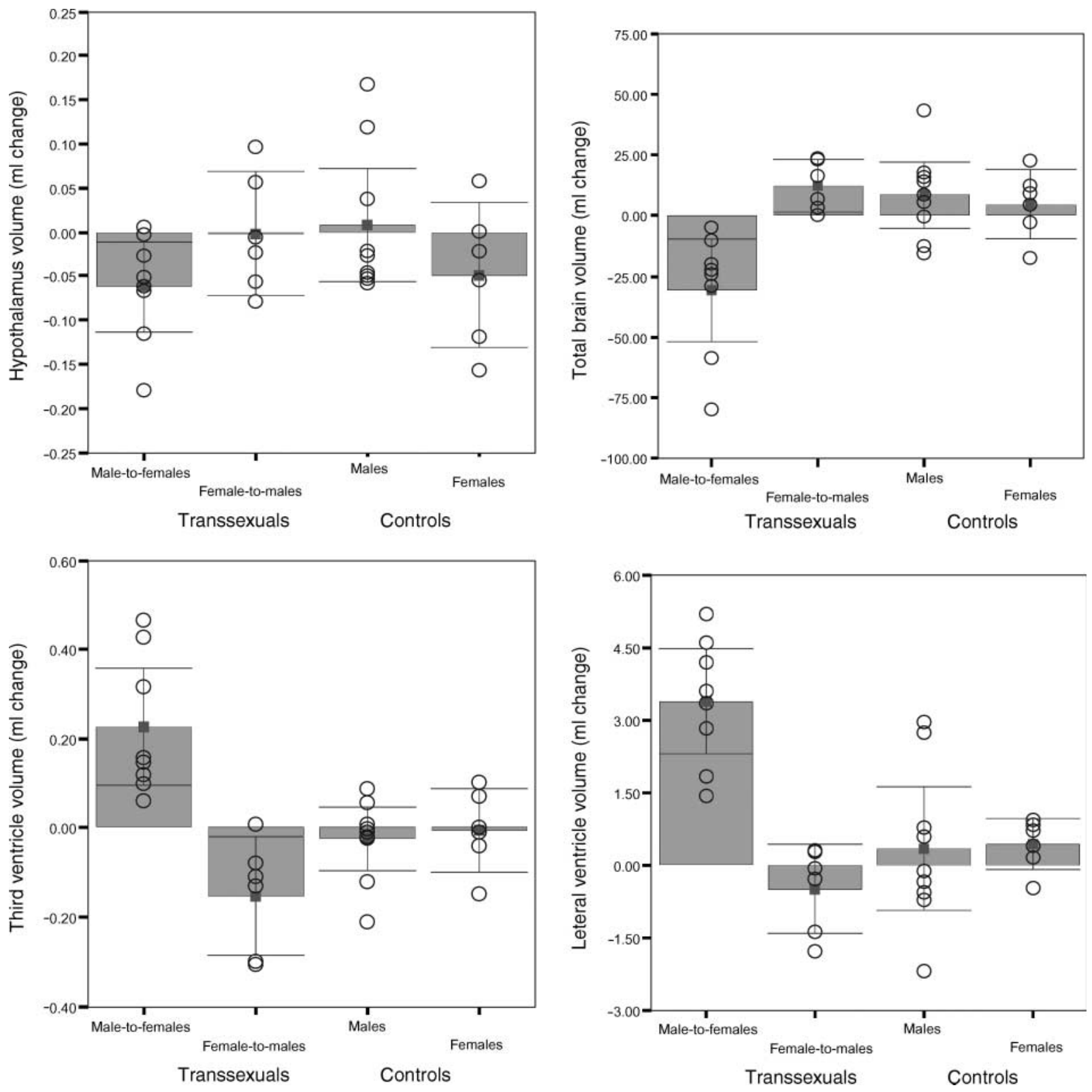

Figure 2 Brain volume changes in male-to-female and female-to-male transsexuals. Changes in hypothalamus, total brain, third and lateral ventricle volumes in milliliters (mean change from 0 at first measurement in bars, $95 \%$ confidence interval in error bars, individual values in circles) in transsexuals following cross-sex hormone treatment as compared with age- and sex-matched healthy comparison subjects.

structures surrounding these ventricles (including, but not limited to, the hypothalamus, which lies in close proximity to the third ventricle). Considering that the effects were not specific for gray (neurons, glia) or white (myelinated axonal fibers) matter suggests that both alterations in nerve cells as well as in axonal fibers may be implicated in the anatomical brain changes following cross-sex hormone treatment in humans. It is not surprising that the influences of sex hormones on the brain were not limited to the hypothalamus, but were also expressed as changes in total brain size. Estrogen and androgen receptor mRNA containing neurons are not limited to the hypothalamus, but are distributed throughout the adult human brain (18).

Prior to cross-sex hormone treatment, no differences in brain volumes between transsexuals and comparison subjects were found. Transsexuals had brain volumes in agreement with their sex at birth. The intracranial volume (and hence overall brain size) and the hypothalamus volume of males were larger than the females, irrespective of the condition of transsexualism. This supports the notion that brain volume changes in 

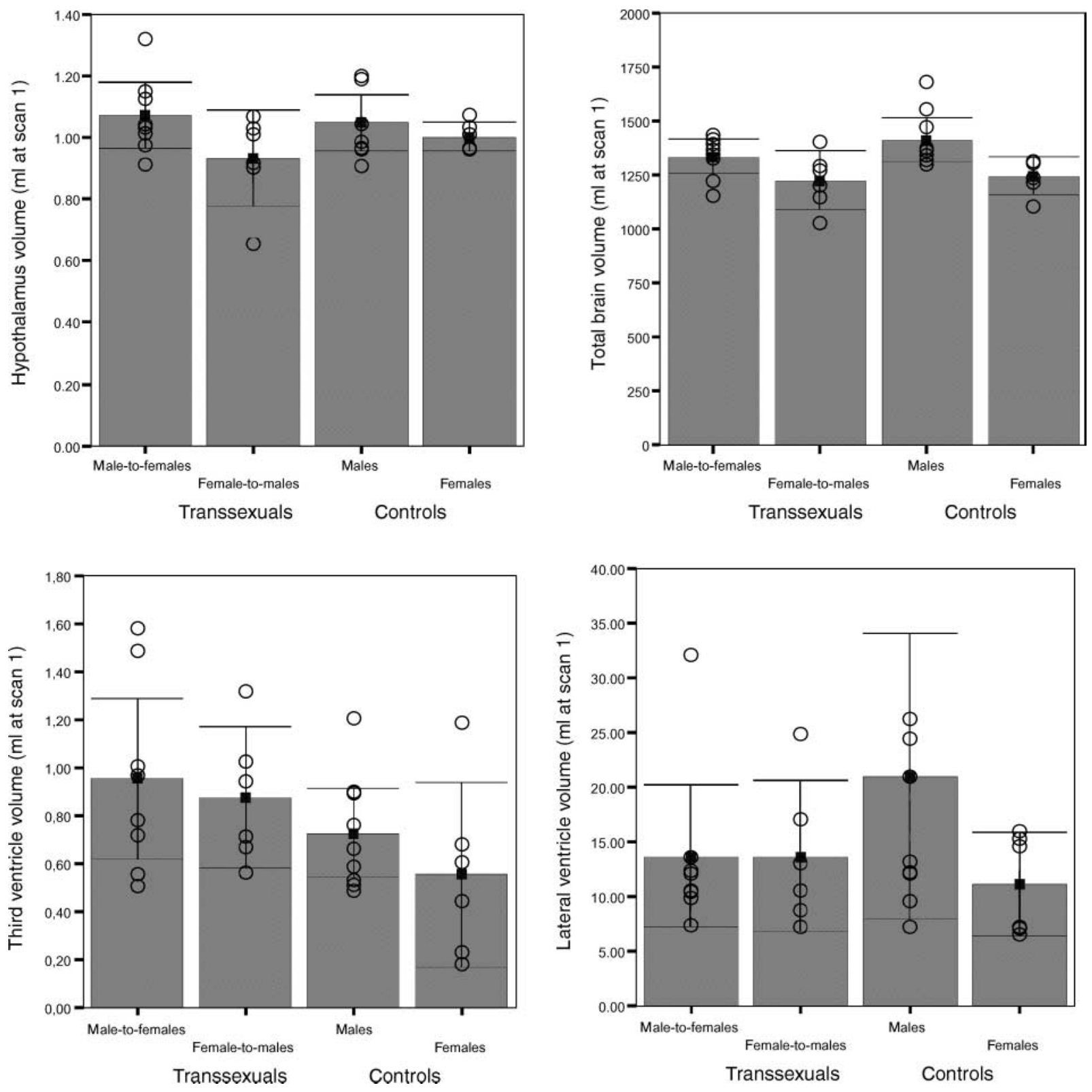

Figure 3 Brain volumes in male-to-female and female-to-male transsexuals just prior to treatment. Hypothalamus, total brain, third and lateral ventricle volumes at scan 1 in milliliters (mean volumes in bars, 95\% confidence interval in error bars, individual values in circles) in transsexuals as compared with age- and sex-matched healthy comparison subjects (individual value of one control male's lateral ventricle $(62 \mathrm{ml})$ not printed).

transsexuals are related to cross-sex hormone treatment and do not reflect pre-existent differences in brain volume between transsexuals and comparisons originating from earlier (pre- or perinatal) brain development. Thus, our findings imply plasticity of adult human brain structure to develop towards the size of the opposite sex under the influence of cross-sex hormones.

Our findings of plastic changes in the total brain volume and hypothalamus volume of transsexuals after cross-sex hormone treatment are corroborated by the observation of (in vitro) structural and functional brain changes in animals upon hormonal manipulations. Since the first study on the reversal of natural sex differences in neuronal connectivity following testosterone hormone treatment in the developing rat (19), numerous studies have documented the influence of sex hormones on brain structure. These include volumetric studies on the influence of cross-sex hormone treatment in adult animals. In the medial preoptic nucleus of the 
hypothalamus (20) of adult gonadectomized male rats, cross-sex hormone treatment (consisting of combined estrogen/progesterone treatment) resulted in significant volume decreases towards female proportions. In addition, in the adult female canary, testosterone triggered the growth of brain vocal control nuclei towards male proportions (21). Moreover, adult castration of male rats and androgen treatment in adult female rats resulted in the reversal of the sexual dimorphism in the medial amygdala (22). Thus, the brain volume changes towards the proportions of the opposite sex observed in the transsexuals following treatment with cross-sex hormones are in agreement with studies in adult animals treated with cross-sex hormones.

Interestingly, in adult female canaries, testosterone treatment also resulted in singing behavior normally heard only from male canaries $(23,24)$. This implies that the sex hormone-induced plastic changes in brain anatomy may be functionally relevant. Indeed, in transsexuals, cross-sex hormone treatment induces changes in behavior and cognitive performance on tests that are known to manifest differences between males and females. In MFs, 3 months of estrogen addition and testosterone suppression resulted in a decline in anger and aggression proneness, sexual arousal, sexual desire, and spatial ability (usually males outperform females) and in an increase in verbal fluency (usually females outperform males) (24, 26, 27). In FMs, 3 months of testosterone treatment was associated with an increase in aggression proneness, sexual arousal, and spatial ability performance, whereas it had a deteriorating effect on verbal fluency tasks $(25,28)$. These behavioral and cognitive findings in transsexuals following cross-sex hormone treatment are in line with the studies reporting influences of endogenous and exogenous sex hormones on behavior and cognition, as well as on cortical brain activation, in non-transsexual adult humans $(29,30)$. Whether functional brain changes accompany the anatomical brain changes in transsexuals remains to be elucidated using functional brain imaging techniques.

Biological mechanisms underlying the gonadal hormone-related plasticity changes in young adult human brains are not known. However, we know that sex steroids have much in common with neurotropins. For instance, like neurotropins, they regulate cell death. Indeed, the most important mechanism by which steroid hormones alter neuron number in sexually dimorphic regions is by influencing cell death. In addition, they are involved in neuronal migration, neurogenesis, and neurotransmitter plasticity. Moreover, these hormones direct formation of sex-specific neuronal networks by influencing axonal guidance and synaptogenesis $(1,31)$. Testosterone treatment in the adult female canary also induces newly activated and expanded vasculature in the vocal control nucleus, which substantially increases the production and release of BDNF; BDNF is both spatially and temporally associated with the recruitment of new neurons (32). Functionally, sex hormones can alter neuronal excitability in a sex-specific way (33). The changes in excitability may be caused not only by altered electrical properties of the membrane, but may also be caused by alternations in neuronal morphology (34). Thus, our reported volume changes in the brains of transsexuals following cross-sex hormone treatment may represent alterations in neuronal cell numbers or the number of synapses. However, more work is needed to uncover particular cells and specific genes on which testosterone (and estrogen) act to regulate cell death in the central nervous system (35).

In conclusion, our data show that in young adult humans, androgen treatment increases the volume of the female brain towards male proportions and antiandrogen + estrogen treatment reduces the size of the male brain towards female proportions. The findings imply plasticity of adult human brain structure towards the opposite sex under the influence of cross-sex hormones.

\section{Acknowledgements}

The authors thank the coordinator of the gender team J Megens at the VU University Medical Center in Amsterdam for his support with patient recruitment and Dr E Fliers for comments on an earlier version of the manuscript.

\section{References}

1 Simerly RB. Wired for reproduction: organization and development of sexually dimorphic circuits in the mammalian forebrain. Annal Reviews of Neuroscience 200225 507-536.

2 Goldstein JM, Seidman LJ, Horton NJ, Makris N, Kennedy DN, Caviness VS Jr, Faraone SV \& Tsuang MT. Normal sexual dimorphism of the adult human brain assessed by in vivo magnetic resonance imaging. Cerebral Cortex 200111 490-497.

3 Swaab DF \& Fliers E. A sexually dimorphic nucleus in the human brain. Science 1985228 1112-1115.

4 Gur RC, Turetsky BI, Matsui M, Yan M, Bilker W, Hughett P \& Gur RE. Sex differences in brain gray and white matter in healthy young adults: correlations with cognitive performance. Journal of Neuroscience 199915 4065-4072.

5 Lemaitre H, Crivello F, Grassiot B, Alperovitch A, Tzourio C \& Mazoyer B. Age- and sex-related effects on the neuroanatomy of healthy elderly. Neuroimage 200526 900-911.

6 De Vries GJ \& Simerly RB. In Hormones, Brain and Behavior, pp 137-191. Eds DW Pfaff, AP Arnold, AM Etgen, SE Fahrbach, RL Moss \& RT Rubin, San Diego: Academic Press, 2002.

7 Breedlove SM \& Hampson E. Sexual differentiation of the brain and behavior. In Behavioral Endocrinology, pp 75-114. Eds JB Becker, SM Breedlove, D Crews \& MM McCarthy, MIT Press: Cambridge, 2002

8 Zhou JN, Hofman MA, Gooren LJ \& Swaab DF. A sex difference in the human brain and its relation to transsexuality. Nature 1995 378 68-70. 
9 Kruijver FP, Zhou JN, Pool CW, Hofman MA, Gooren LJ \& Swaab DF. Male-to-female transsexuals have female neuron numbers in a limbic nucleus. Journal of Clinical Endocrinolology and Metabolism 200085 2034-2041.

10 Agate RJ, Grisham W, Wade J, Mann S, Wingfield J, Schanen C, Palotie A \& Arnold AP. Neural, not gonadal, origin of brain sex differences in a gynandromorphic finch. PNAS $2003 \mathbf{1 0 0}$ 4873-4878.

11 Spitzer RL, Williams JB, Gibbon M \& First MB. The structured clinical interview for DSM-III-R (SCID). I: History, rationale, and description. Archives of General Psychiatry $199249624-629$.

12 Cohen-Kettenis PT \& Gooren LJG. Transsexualism: a review of etiology, diagnosis and treatment. Journal of Psychosomatic Research 199946 315-333.

13 Schnack HG, Hulshoff Pol HE, Baare WF, Staal WG, Viergever MA \& Kahn RS. Automated separation of gray and white matter from MR images of the human brain. Neuroimage 200113 230-237.

14 Schnack HG, Hulshoff Pol HE, Baare WF, Viergever MA \& Kahn RS. Automatic segmentation of the ventricular system from MR images of the human brain. Neuroimage 2001 14 95-104.

15 Nieuwenhuys R, Voogd J \& Van Huijzen C. The Human Central Nervous System. edn 3. Berlin: Springer-Verlag, 1988.

16 Hulshoff Pol HE, Schnack HG, Bertens MG, van Haren NEM, van der Tweel I, Staal WG, Baare WF \& Kahn RS. Gray matter volume changes in schizophrenia. American Journal of Psychiatry 2002 $159244-250$

17 Liu RS, Lemieux L, Bell GS, Sisodiya SM, Shorvon SD, Sander JW \& Duncan JS. A longitudinal study of brain morphometrics using quantitative magnetic resonance imaging and difference image analysis. Neuroimage $20032022-33$.

18 Simerly RB, Chang C, Muramatsu M \& Swanson LW. Distribution of androgen and estrogen receptor mRNA-containing cells in the rat brain: an in situ hybridization study. Journal of Comparative Neurology 1990294 76-95.

19 Raisman G \& Field PM. Sexual dimorphism in the preoptic area of the rat. Science $1971173731-733$.

20 Bloch GJ \& Gorski RA. Estrogen/progesterone treatment in adulthood affects the size of several components of the medial preoptic area in the male rat. Journal of Comparative Neurology 1988275 613-622.

21 Nottebohm F. Testosterone triggers growth of brain vocal control nuclei in adult female canaries. Brain Research 1980189429.

22 Cooke BM, Tabibnia G \& Breedlove SM. A brain sexual dimorphism controlled by adult circulating androgens. PNAS $1999967538-7540$.

23 DeVoogd T \& Nottebohm F. Gonadal hormones induce dendritic growth in the adult avian brain. Science $1981 \mathbf{2 1 4} 202-204$.
24 Tramontin AD \& Brenowitz EA. Seasonal plasticity in the adult brain. Trends in Neuroscience 200023 251-258.

25 Van Goozen SHM, Cohen-Kettenis PT, Gooren LJG, Frijda NH \& Van der Pol NE. Gender differences in behavior: activating effects of cross-sex hormones. Psychoneuroendocrinology 1995 20 343-363.

26 Van Kemenade JF, Cohen-Kettenis PT, Cohen L \& Gooren LJ. Effects of the pure antiandrogen RU 23.903 (anandron) on sexuality, aggression and mood in male-to-female transsexuals. Archives of Sexual Behavior 198918 217-218.

27 Van Goozen SHM, Slabbekoorn D, Gooren LJG, Sanders G \& Cohen-Kettenis PT. Organizing and activating effects of sex hormones in homosexual transsexuals. Behavioral Neuroscience $2002116982-988$.

28 Van Goozen SHM, Cohen-Kettenis PT, Gooren CJG, Frijda NH \& Van der Pol NE. Activa-ting effects of angrogens on cognitive performance: causal evidence in a group of female-to-male transsexuals. Neuropsychologia 199432 1153-1157.

29 Hampson E. Sex differences in human brain and cognition. In Behavioral Endocrinology, edn 2, pp 579-630. Eds JB Becker, SM Breedlove, D Crews \& MM McCarthy, Cambridge: MIT Press, 2002.

30 Fernandez G, Weis S, Stoffel-Wagner B, Tendolkar I, Reuber M, Beyenburg S, Klaver P, Fell J, de Greiff A, Ruhlmann J, Reul J \& Elger CE. Menstrual cycle-dependent neural plasticity in the adult human brain is hormone, task, and region specific. Journal of Neuroscience 200323 3790-3795.

31 Kawata M. Roles of steroid hormones and their receptors in structural organization in the nervous system. Neuroscience Research $1995 \mathbf{2 4} 1-46$

32 Louissaint A Jr, Rao S, Leventhal C \& Goldman SA. Coordinated interaction of neurogenesis and angiogenesis in the adult songbird brain. Neuron 200234 945-960.

33 Teyler TJ, Vardaris RM, Lewis D \& Rawitch AB. Gonadal steroids: effects on excitability of hipppocampal pyramidal cells. Science $19802091017-1019$.

34 Joëls M. Steroid hormones and excitability in the mammalian brain. Frontiers in Neuroendocrinology 199718 2-48.

35 Morris JA, Jordan CL \& Breedlove SM. Sexual differentiation of the vertebrate nervous system. Naure Neuroscience 200410 1034-1039.

Received 30 April 2006

Accepted 7 July 2006 